Research Article

\title{
Vibration Power Flow of an Infinite Cylindrical Shell Submerged in Viscous Fluids
}

\author{
Haosen Chen, ${ }^{1}$ Peng Yang $\mathbb{D}^{2},{ }^{2}$ and Yijun Shen ${ }^{1}$ \\ ${ }^{1}$ School of Civil Engineering and Architecture, Hainan University, Haikou 570228, China \\ ${ }^{2}$ School of Mechanics and Electrics Engineering, Hainan University, Haikou 570228, China \\ Correspondence should be addressed to Peng Yang; pycssrc@qq.com
}

Received 2 June 2020; Revised 20 August 2020; Accepted 31 August 2020; Published 12 September 2020

Academic Editor: Anindya Ghoshal

Copyright ( 2020 Haosen Chen et al. This is an open access article distributed under the Creative Commons Attribution License, which permits unrestricted use, distribution, and reproduction in any medium, provided the original work is properly cited.

In the previous investigations of the vibroacoustic characteristics of a submerged cylindrical shell in a flow field, the fluid viscosity was usually ignored. In this paper, the effect of fluid viscosity on the characteristics of vibration power flow in an infinite circular cylindrical shell immersed in a viscous acoustic medium is studied. Flügge's thin shell theory for an isotropic, elastic, and thin cylindrical shell is employed to obtain the motion equations of the structure under circumferential-distributed line force. Together with the wave equations for the viscous flow field as well as continuity conditions at the interface, the vibroacoustic equation of motion in the coupled system is derived. Numerical analysis based on the additional-damping numerical integral method and tenpoint Gaussian integral method is conducted to solve the vibroacoustic coupling equation with varying levels of viscosity. Then, the variation of the input power flow against the nondimensional axial wave number in the coupled system with different circumferential mode numbers is discussed in detail. It is found that the influence of fluid viscosity on the vibroacoustic coupled system is mainly concentrated in the low-frequency band, which is shown as the increase of the crest number and amplitude of the input power flow curves.

\section{Introduction}

Much research has been conducted on the analysis of the vibroacoustic characteristics of an infinite elastic thin cylindrical shell submerged in an acoustic medium. One of the earliest was done by Junger, who found that the existence of entrained water could greatly reduce the natural frequency of the shell due to added-mass effects and showed that the dynamic behavior of the structure was mainly affected in the low-frequency band $[1,2]$. Recently, Guo et al. studied the sound-structure coupling problem of a cylindrical shell submerged in a quarter water domain and found that the complex acoustic boundary has a crucial influence on the vibration of the cylindrical shell when the cylindrical shell approaches the boundary while the influence tends to vanish when the distances between the cylindrical shell and the boundaries exceed certain values [3]. Guo et al. investigated the far-field acoustic radiation of a cylindrical shell with finite length submerged at a finite depth from the water surface, which showed that the vibration of the cylindrical shell submerged at a finite depth from the free surface tends to be the same as that in infinite fluid when the submerged depth exceeds a certain value. Besides, the frequency and the submerged depth have crucial effects on the fluctuation of the far-field sound pressure [4]. Zhang et al. discussed the free vibration characteristics of an eccentric cylindrical shell and suggested that there are symmetrical and antisymmetric modes with different frequencies and modes, and the natural frequency of eccentric cylindrical shell decreases with the increase of eccentricity [5]. Miao et al. studied the acoustical radiation characteristics of a cylindrical shell in a shallow sea and found that the sound absorption is apparently different from the radiative sound field of the cylindrical shell under the condition of a rigid seafloor [6].

It should be noted that the surrounding fluid was generally considered to be inviscid in the previous research. However, fluid viscosity is inevitable in practice and the ignorance of its influence on the vibroacoustic behavior of 
the submerged cylindrical shells may result in large inaccuracy in certain circumstances.

Amabili and Garziera investigated the effect of steady viscous forces on vibrations of shells with nonuniform constraints, added masses, elastic bed, and prestress conveying or immersed in annular axial flow and found that the effect of viscous loads can be considered stabilizing if compared with results computed for shells conveying inviscid flow having the same outlet pressure. Besides, the contrary is obtained if a comparison is made with the results computed for shells having the same inlet pressure [7]. These conclusions were instructive despite the fact that the flow field was considered incompressible. Su studied the axisymmetric free oscillations of a fluid-filled spherical shell immersed in a sound field and came to the conclusion that both the damping of the shell motion and the acoustic radiation damping are reduced by fluid viscosity since the viscous dissipation damping must remain positive $[8,9]$. Moreover, Chen et al. found that fluid viscosity affected the axial wave number and the cut-on and cut-off frequencies of the propagating waves when studying the dispersion characteristics of an immersed cylindrical shell considering hydrostatic pressure [10]. Sorokin and Chubinskij addressed free wave propagation and attenuation in an elastic conventional Kirchhoff plate and in a Sandwich plate loaded by a quiescent viscous compressible fluid, respectively, and demonstrated that the characteristics of each wave in the coupled system were affected considering fluid viscosity [11].

Therefore, considerations of the influence of fluid viscosity can better reflect reality and thus provide a more accurate theoretical model. There is also little work done regarding the vibroacoustic characteristics of an infinite circular cylindrical shell submerged in viscous fluids.

As an attempt to address these issues, this paper investigates the vibroacoustic characteristics of an isotropic, elastic, and thin cylindrical shell coupled with compressible fluids taking into account the viscosity. The acoustic wave equations of the coupled system considering hydrostatic pressure are derived based on Flügge's thin shell theory and the linearized equations of continuity as well as the Navier-Stokes equations. Then, the pertinent boundary conditions are employed to obtain the vibroacoustic coupled equation of motion of the coupled system under circumferential-distributed line force. After that, the numerical algorithms are developed to solve the equations with consideration of varying fluid viscosity to obtain the curves of the input power flow versus circular frequency with different circumferential mode numbers. Based on the results, the influence of fluid viscosity on the vibroacoustic characteristics of the coupled system is analyzed.

\section{The Governing Equations}

The geometry and the coordinate system are shown in Figure 1. The dynamic problem of the coupled system is formulated in a cylindrical polar coordinate system $(x, \theta, r)$. The coordinate axis $x$ is chosen to coincide with the cylindrical shell centerline while the coordinate axes $r$ and $\theta$ are taken along the radial and circumferential directions, respectively.

Assume that a circular cosine-distributed harmonic force is applied at the section $x=0$ of the cylindrical shell, and it is expressed as follows:

$$
F(\theta, t)=F_{0} \cos n \theta \delta(0) \exp (i \omega t) .
$$

2.1. Shell Equations. It is assumed that the material of the thin cylindrical shell is isotropic and elastic, and the thickness of the shell is much smaller compared with its mean radius. The governing equations of motion are obtained based on Flügge's thin shell theory, which can be written as [12]

$$
\left\{\begin{array}{l}
U_{x x}+\frac{1-v}{2} U_{\theta \theta}+\frac{1+v}{2} V_{x \theta}+\nu W_{x}+K\left(\frac{1-v}{2} U_{\theta \theta}-W_{x x x}+\frac{1-v}{2} W_{x \theta \theta}\right)-\frac{\rho_{s} R^{2}\left(1-v^{2}\right)}{E} U_{t t}=-\frac{R^{2}\left(1-v^{2}\right)}{E h} P_{x}, \\
\frac{1+v}{2} U_{x \theta}+\frac{1-v}{2} V_{x x}+V_{\theta \theta}+\left(1+T_{2}\right) W_{\theta}+K\left[\frac{3(1-v)}{2} V_{x x}-\frac{3-v}{2} W_{x x \theta}\right]-\frac{\rho_{s} R^{2}\left(1-v^{2}\right)}{E} V_{t t}=-\frac{R^{2}\left(1-v^{2}\right)}{E h} P_{\theta}, \\
v U_{x}-K U_{x x x}+K \frac{1-v}{2} U_{x \theta \theta}+V_{\theta}-K \frac{3-v}{2} V_{x x \theta}+(1+K) W+K W_{x x x x}+2 K W_{x x \theta \theta}+K W_{\theta \theta \theta \theta}+2 K W_{\theta \theta}+\frac{\rho_{s} R^{2}\left(1-v^{2}\right)}{E} W_{t t}=-\frac{R^{2}\left(1-v^{2}\right)}{E h} P_{r}+F,
\end{array}\right.
$$

where ()$_{x}=R(\partial() / \partial x),()_{\theta}=(\partial() / \partial \theta),()_{t}=(\partial() / \partial t), K=$ $\left(h^{2} / 12 R^{2}\right)$.

Using a wave propagation approach, the solution of equation (2) takes the form of standing waves when the shell vibrates. Considering that the vibration wave propagates along the axial direction of the shell, the displacement components can be expanded into normal wave-forms related to its axial wave numbers:

$$
\left\{\begin{array}{l}
U(x, \theta, r, t)=\sum_{n=0}^{\infty} \sum_{s=1}^{\infty} U_{n s} \cos n \theta \exp \left(i \omega t-i k_{n s} x\right), \\
V(x, \theta, r, t)=\sum_{n=0}^{\infty} \sum_{s=1}^{\infty} V_{n s} \sin n \theta \exp \left(i \omega t-i k_{n s} x\right), \\
W(x, \theta, r, t)=\sum_{n=0}^{\infty} \sum_{s=1}^{\infty} W_{n s} \cos n \theta \exp \left(i \omega t-i k_{n s} x\right) .
\end{array}\right.
$$




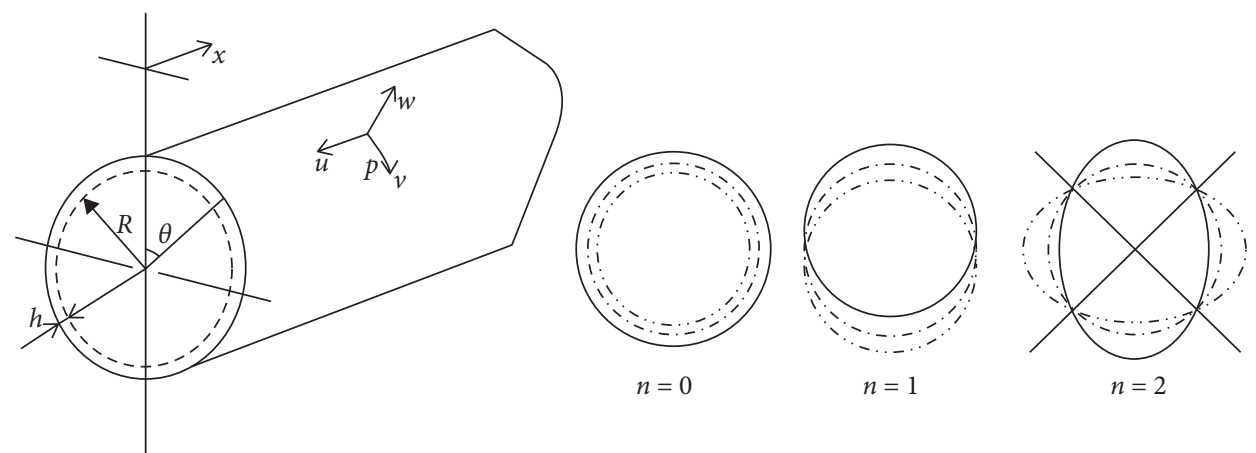

FIgURE 1: Coordinate system and circumferential mode shapes.

Substituting equation (3) into equation (2) results in the shell equation of motion:

$$
\left[\begin{array}{ccc}
L_{11} & L_{12} & L_{13} \\
L_{21} & L_{22} & L_{23} \\
L_{31} & L_{32} & L_{33}
\end{array}\right]\left[\begin{array}{c}
U_{n s} \\
V_{n s} \\
W_{n s}
\end{array}\right]=-\frac{R^{2}\left(1-v^{2}\right)}{E h}\left[\begin{array}{c}
P_{x} \\
P_{\theta} \\
P_{r}-F
\end{array}\right],
$$

where $L_{11}=\Omega^{2}-\lambda^{2}-((1+K)(1-v) / 2) n^{2}, \quad L_{12}=-($ in $\lambda$ $(1+\nu) / 2)=L_{21}, \quad L_{13}=-i\left[\nu \lambda+K \lambda^{3}-\left(K(1-\nu) n^{2} \lambda / 2\right)\right]=$ $L_{31}, L_{22}=n^{2}-((1+3 K)(1-v) / 2) \lambda^{2}+\Omega^{2}, L_{23}=-n-((3-v)$ $\left.K n \lambda^{2} / 2\right)=-L_{32}, \quad L_{33}=1+K+K \lambda^{4}+2 K n^{2} \lambda^{2}+K n^{4}-K n^{2}-$ $\Omega^{2} ; \Omega=\omega \sqrt{\rho_{s} R^{2}\left(1-v^{2}\right) / E}, \lambda=k_{n s} R$.

2.2. Fluid Acoustics Equations. The fluid is assumed to be viscous, nonheat-conducting, compressible, and isotropic and, thus, the acoustic wave equation is valid. The linearized equation of continuity is

$$
\frac{\partial \rho_{f}}{\partial t}+\rho_{f} \nabla \cdot \mathbf{u}_{f}=0
$$

And the Navier-Stokes equation is

$$
\rho_{f} \frac{\mathrm{D} \mathbf{u}_{f}}{\mathrm{D} t}=-\nabla p+\mu \nabla^{2} \mathbf{u}_{f}+\left(\frac{\mu}{3}+\mu_{b}\right) \nabla\left(\nabla \cdot \mathbf{u}_{f}\right) .
$$

For a barotropic fluid, the linear equation of state is

$$
\frac{\partial p}{\partial t}=c_{f}^{2} \frac{\partial \rho_{f}}{\partial t} .
$$

Wave propagation and the consequent vibration characteristics are focused on in this paper, and the velocity vector $\mathbf{u}_{f}$ at any point in the flow field is much smaller compared to the wave and sound velocity $c_{\mathrm{f}}$. Assuming the dimension of $\mathbf{u}_{f}$ is $\varepsilon$, the dimension of the product of any term involving fluid velocity components is $\varepsilon^{2}$. Therefore, the product of any term involving fluid velocity components can be regarded as an infinitesimal of a higher order. With infinitesimals of higher order ignored, the total differential terms in the Navier-Stokes equation can be simplified, such as

$$
\begin{aligned}
\frac{\mathrm{D} u_{f x}}{\mathrm{D} t} & =\frac{\partial u_{f x}}{\partial t}, \\
\frac{\partial\left(\mathrm{D} u_{f x} / \mathrm{D} t\right)}{\partial t} & =\frac{\partial^{2} u_{f x}}{\partial t^{2}} .
\end{aligned}
$$

Therefore, a partial derivative about $t$ on both sides of equation (6) results in

$$
\frac{\partial \rho_{f}}{\partial t} \frac{\partial \mathbf{u}_{f}}{\partial t}+\rho_{f} \cdot \frac{\partial^{2} \mathbf{u}_{f}}{\partial t^{2}}=-\nabla\left(\frac{\partial p}{\partial t}\right)+\mu \nabla^{2}\left(\frac{\partial \mathbf{u}_{f}}{\partial t}\right)+\left(\frac{\mu}{3}+\mu_{b}\right) \nabla\left(\nabla \cdot \frac{\partial \mathbf{u}_{f}}{\partial t}\right) .
$$

Then, equations (5), (7), and (9) can be combined to yield

$$
-\rho_{f}\left(\nabla \cdot \mathbf{u}_{f}\right) \frac{\partial \mathbf{u}_{f}}{\partial t}+\rho_{f} \cdot \frac{\partial^{2} \mathbf{u}_{f}}{\partial t^{2}}=c_{f}^{2} \rho_{f} \nabla\left(\nabla \cdot \mathbf{u}_{f}\right)+\mu \nabla^{2}\left(\frac{\partial \mathbf{u}_{f}}{\partial t}\right)+\left(\frac{\mu}{3}+\mu_{b}\right) \nabla\left(\nabla \cdot \frac{\partial \mathbf{u}_{f}}{\partial t}\right)
$$

Ignoring the higher-order terms in equation (10) leads to a single equation for the velocity vector $\mathbf{u}_{f}$, which is linearized [13]:

$$
\frac{\partial^{2} \mathbf{u}_{f}}{\partial t^{2}}-c_{f}^{2} \nabla\left(\nabla \cdot \mathbf{u}_{f}\right)=\frac{\mu}{\rho_{f}} \nabla^{2} \frac{\partial \mathbf{u}_{f}}{\partial t}+\frac{1}{\rho_{f}}\left(\frac{\mu}{3}+\mu_{b}\right) \nabla\left(\nabla \cdot \frac{\partial \mathbf{u}_{f}}{\partial t}\right) .
$$

According to the Helmholtz decomposition theorem, the velocity fields can be resolved as the superposition of longitudinal and transverse vector components:

$$
\mathbf{u}_{f}=\nabla \phi+\nabla \times \psi \text {. }
$$

Introducing the above decomposition into equation (11) and making use of the symmetry condition of the system, 
$\psi=(0,0, \psi)$, as well as the calibration condition, $\nabla \cdot \psi=0$, a set of two equations is deduced:

$$
\left\{\begin{array}{l}
\frac{\partial^{2} \phi}{\partial t^{2}}=\left[c_{f}^{2}+\frac{1}{\rho_{f}}\left(\frac{4}{3} \mu+\mu_{b}\right) \frac{\partial}{\partial t}\right] \nabla^{2} \phi, \\
\frac{\partial \psi}{\partial t}=\frac{\mu}{\rho_{f}} \nabla^{2} \psi .
\end{array}\right.
$$

If the wave is assumed to be monochromatic, the solutions are expected to be of the following form [14]:

$$
\left\{\begin{array}{l}
\phi(r, x, \theta, t)=\phi(r, x, \theta, \omega) \exp (i \omega t), \\
\psi(r, x, \theta, t)=\psi(r, x, \theta, \omega) \exp (i \omega t) .
\end{array}\right.
$$

Incorporate the assumptions above in equation (13), and yield the following equation after some manipulations:

$$
\left\{\begin{array}{l}
\left(\nabla^{2}+k_{c}^{2}\right) \phi=0, \\
\left(\nabla^{2}+k_{s}^{2}\right) \psi=0,
\end{array}\right.
$$

where $k_{c}=(\omega / c)\left[1-i\left(\omega \mu / 2 \rho c^{2}\right)\left((4 / 3)+\left(\mu_{b} / \mu\right)\right)\right], k_{s}=(1-$ i) $\sqrt{\omega \rho / 2 \mu}$.

In the cylindrical coordinate, the solutions can be expressed as follows $[15,16]$ :

$$
\left\{\begin{array}{l}
\phi(x, \theta, r, t)=\sum_{n=0}^{\infty} \sum_{s=1}^{\infty} A_{n s} H_{n}^{(2)}\left(\gamma_{c} r\right) \cos n \theta \exp \left(i \omega t-i k_{n s} x\right), \\
\psi_{x}(x, \theta, r, t)=\sum_{n=1}^{\infty} \sum_{s=1}^{\infty} B_{n s} H_{n}^{(2)}\left(\gamma_{s} r\right) \sin n \theta \exp \left(i \omega t-i k_{n s} x\right), \\
\psi_{\theta}(x, \theta, r, t)=-\sum_{n=0}^{\infty} \sum_{s=1}^{\infty} C_{n s} H_{n+1}^{(2)}\left(\gamma_{s} r\right) \cos n \theta \exp \left(i \omega t-i k_{n s} x\right), \\
\psi_{r}(x, \theta, r, t)=\sum_{n=1}^{\infty} \sum_{s=1}^{\infty} C_{n s} H_{n+1}^{(2)}\left(\gamma_{s} r\right) \sin n \theta \exp \left(i \omega t-i k_{n s} x\right),
\end{array}\right.
$$

where $\gamma_{c}=\sqrt{k_{c}^{2}-k_{n s}^{2}}, \gamma_{s}=\sqrt{k_{s}^{2}-k_{n s}^{2}}$.

Now, considering the basic field equations in cylindrical coordinates, the velocity components of the waves in $x-, \theta-$, and $r$-direction in terms of potentials in the viscous fluid are [17]

$$
\left\{\begin{array}{l}
u_{x}=-\frac{\partial \phi}{\partial x}+\frac{1}{r} \frac{\partial\left(r \psi_{\theta}\right)}{\partial r}-\frac{1}{r} \frac{\partial \psi_{r}}{\partial \theta} \\
u_{\theta}=-\frac{1}{r} \frac{\partial \phi}{\partial \theta}+\frac{\partial \psi_{r}}{\partial x}-\frac{\partial \psi_{x}}{\partial r}, \\
u_{r}=-\frac{\partial \phi}{\partial r}+\frac{1}{r} \frac{\partial \psi_{x}}{\partial \theta}-\frac{\partial \psi_{\theta}}{\partial x}
\end{array}\right.
$$

Similarly, the stress components in the fluid are [18]

$$
\left\{\begin{array}{l}
\sigma_{r x}=\mu\left(\frac{\partial u_{r}}{\partial x}+\frac{\partial u_{x}}{\partial r}\right), \\
\sigma_{r \theta}=\mu\left(\frac{1}{r} \frac{\partial u_{r}}{\partial \theta}+\frac{\partial u_{\theta}}{\partial r}-\frac{u_{\theta}}{r}\right), \\
\sigma_{r r}=-i \omega \rho \phi+2 \mu k_{c}^{2} \phi+2 \mu \frac{\partial u_{r}}{\partial r} .
\end{array}\right.
$$

With the harmonic terms ignored, a substitution of equation (16) into equations (17) and (18) results in

$$
\begin{gathered}
\left\{\begin{array}{l}
u_{x}=\frac{\cos n \theta}{r}\left\{i k_{n s} r H_{n}^{(2)}\left(\gamma_{c} r\right) A_{n s}-\left[(n+1) H_{n+1}^{(2)}\left(\gamma_{s} r\right)+\gamma_{s} r H_{n+1}^{(2)^{\prime}}\left(\gamma_{s} r\right)\right] C_{n s}\right\}, \\
u_{\theta}=\frac{\sin n \theta}{r}\left[n H_{n}^{(2)}\left(\gamma_{c} r\right) A_{n s}-\gamma_{s} r H_{n+1}^{(2)^{\prime}}\left(\gamma_{s} r\right) B_{n s}-i k_{n s} r H_{n+1}^{(2)}\left(\gamma_{s} r\right) C_{n s}\right], \\
u_{r}=-\frac{\cos n \theta}{r}\left[\gamma_{c} r H_{n}^{(2))^{\prime}}\left(\gamma_{c} r\right) A_{n s}-n H_{n}^{(2)}\left(\gamma_{s} r\right) B_{n s}+i k_{n s} r H_{n+1}^{(2)}\left(\gamma_{s} r\right) C_{n s}\right],
\end{array}\right. \\
\left\{\begin{array}{l}
\sigma_{r x}=\frac{\mu}{r^{2}} \cos n \theta\left\{2 i k_{n s} \gamma_{c} r^{2} H_{n}^{(2)^{\prime}}\left(\gamma_{c} r\right) A_{n s}-i n k_{n s} r H_{n}^{(2)}\left(\gamma_{s} r\right) B_{n s}+\left[\left(k_{n s}^{2} r^{2}+n+1\right) H_{n+1}^{(2)}\left(\gamma_{s} r\right)-(n+1) \gamma_{s} r H_{n+1}^{(2)^{\prime}}\left(\gamma_{s} r\right)-\gamma_{s}^{2} r^{2} H_{n+1}^{(2)^{\prime}}\left(\gamma_{s} r\right)\right] C_{n s}\right\}, \\
\sigma_{r \theta}=\frac{\mu}{r^{2}} \sin n \theta\left\{2 n\left[-H_{n}^{(2)}\left(\gamma_{c} r\right)+\gamma_{c} r H_{n}^{(2)^{\prime}}\left(\gamma_{c} r\right)\right] A_{n s}-\left[n^{2} H_{n}^{(2)}\left(\gamma_{s} r\right)-\gamma_{s} r H_{n}^{(2)^{\prime}}\left(\gamma_{s} r\right)+\gamma_{s}^{2} r^{2} H_{n}^{(2)^{\prime}}\left(\gamma_{s} r\right)\right] B_{n s}-i k_{n s} r\left[(n+1) H_{n+1}^{(2)}\left(\gamma_{s} r\right)-\gamma_{s} r H_{n+1}^{(2)}\left(\gamma_{s} r\right)\right] C_{n s}\right\}, \\
\sigma_{r r}=\frac{\cos n \theta}{r^{2}}\left\{\left[r^{2}\left(i \omega \rho-2 \mu k_{c}^{2}\right) H_{n}^{(2)}\left(\gamma_{s} r\right)-2 \mu \gamma_{c}^{2} r^{2} H_{n}^{(2)}\left(\gamma_{c} r\right)\right] A_{n s}-2 \mu n\left[H_{n}^{(2)}\left(\gamma_{s} r\right)-\gamma_{s} r H_{n}^{(2)}\left(\gamma_{s} r\right)\right] B_{n s}-2 i \mu k_{n s} r^{2} \gamma_{s} H_{n+1}^{(2)}\left(\gamma_{s} r\right) C_{n s}\right\} .
\end{array}\right.
\end{gathered}
$$


2.3. The Equations of Motion in the Coupled System. The stress components in the fluid derived using the wave propagation approach serve as the equivalent loads on the thin-walled cylindrical shell applied by the medium in the directions of $x, \theta$, and $r$ :

$$
\begin{aligned}
& \left.\sigma_{r x}\right|_{r=R}=P_{x}, \\
& \left.\sigma_{r \theta}\right|_{r=R}=P_{\theta}, \\
& \left.\sigma_{r r}\right|_{r=R}=P_{r},
\end{aligned}
$$

where the dimension of the surface loads after Fourier transform to $P_{x}, P_{\theta}$, and $P_{r}$, rather than the force dimension, is taken.

According to the motion coordination conditions of the outer surface of the shell, the velocities in the three directions must be continuous at the fluid-shell boundary, leading to the continuous boundary conditions:

$$
\begin{aligned}
& \left.u_{x}\right|_{r=R}=\left.\frac{\partial U}{\partial t}\right|_{r=R}, \\
& \left.u_{\theta}\right|_{r=R}=\left.\frac{\partial V}{\partial t}\right|_{r=R}, \\
& \left.u_{r}\right|_{r=R}=\left.\frac{\partial W}{\partial t}\right|_{r=R} .
\end{aligned}
$$

A matrix equation can be obtained by substituting equation (20) into equation (21):

$$
\left[\begin{array}{lll}
P_{x} & P_{\theta} & P_{r}
\end{array}\right]^{T}=\frac{1}{R^{2}}\left[Q_{3 \times 3}\right]\left[\begin{array}{lll}
A_{n s} & B_{n s} & C_{n s}
\end{array}\right]^{T} .
$$

Substituting equation (23) into equation (4) results in

$$
\left[L_{3 \times 3}\right]\left[\begin{array}{lll}
U_{n s} & V_{n s} & W_{n s}
\end{array}\right]^{T}=-\frac{\left(1-v^{2}\right)}{E h}\left[Q_{3 \times 3}\right]\left[\begin{array}{lll}
A_{n s} & B_{n s} & C_{n s}
\end{array}\right]^{T}+\frac{\left(1-v^{2}\right)}{E h}\left[\begin{array}{lll}
0 & 0 & F
\end{array}\right]^{T},
$$

where $Q_{11}=2 i \mu \lambda \gamma_{c} r H_{n}^{(2)}\left(\gamma_{c} R\right), Q_{12}=-i \mu n \lambda H_{n}^{(2)}\left(\gamma_{s} R\right), Q_{13}=$ $\mu\left(\lambda^{2}+n+1\right) H_{n+1}^{(2)} \quad\left(\gamma_{s} R\right)-\mu(n+1) \gamma_{s} R H_{n+1}^{(2)}\left(\gamma_{s} R\right)-$ $\mu \gamma_{s}^{2} R^{2} H_{n+1}^{(2)}\left(\gamma_{s} R\right), \quad Q_{21}=-2 \mu n\left[H_{n}^{(2)}\left(\gamma_{c} R\right)-\gamma_{c} R H_{n}^{(2)}\left(\gamma_{c} R\right)\right]$, $Q_{22}=-\mu\left[n^{2} H_{n}^{(2)}\left(\gamma_{s} R\right)-\gamma_{s} R H_{n}^{(2)}\left(\gamma_{s} R\right)+\gamma_{s}^{2} R^{2} H_{n}^{(2)}\left(\gamma_{s} R\right)\right]$, $Q_{23}=-i \mu \lambda\left[(n+1) H_{n+1}\left(\gamma_{s} R\right)-\gamma_{s} R H_{n+1}^{\prime}\left(\gamma_{s} R\right)\right], \quad Q_{31}=$ $R^{2}\left(i \omega \rho-2 \mu k_{c}^{2}\right) H_{n}\left(\gamma_{c} R\right)-2 \mu \gamma_{c}^{2} R^{2} H_{n}^{\prime \prime}\left(\gamma_{c} R\right), \quad Q_{32}=-2 \mu n$ $\left[H_{n}\left(\gamma_{s} R\right)-\gamma_{s} R H_{n}^{\prime}\left(\gamma_{s} R\right)\right], Q_{33}=-2 i \mu \lambda \gamma_{s} R H_{n+1}^{\prime}\left(\gamma_{s} R\right)$.

Then, the velocity continuous conditions lead to a matrix equation as follows:

$$
\left[\begin{array}{lll}
U_{n s} & V_{n s} & W_{n s}
\end{array}\right]^{T}=-\frac{i R^{2}}{\Omega} \sqrt{\frac{\rho_{s}\left(1-v^{2}\right)}{E}}\left[\begin{array}{lll}
O_{3 \times 3}
\end{array}\right]\left[\begin{array}{lll}
A_{n s} & B_{n s} & C_{n s}
\end{array}\right]^{T},
$$

where $O_{11}=i \lambda H_{n}^{(2)}\left(\gamma_{c} R\right), \quad O_{12}=0, \quad O_{13}=-\left[(n+1) H_{n+1}^{(2)}\right.$ $\left.\left(\gamma_{s} R\right)+\gamma_{s} R H_{n+1}^{(2)}\left(\gamma_{s} R\right)\right], \quad O_{21}=n H_{n}^{(2)}\left(\gamma_{c} R\right), \quad O_{22}=-\gamma_{s}$ $R H_{n+1}^{(2)}\left(\gamma_{s} R\right), \quad O_{23}=-i \lambda H_{n+1}^{(2)}\left(\gamma_{s} R\right), \quad O_{31}=-\gamma_{c} R H_{n}^{(2)}\left(\gamma_{c} R\right)$, $\mathrm{O}_{32}=n H_{n}^{(2)}\left(\gamma_{s} R\right), \mathrm{O}_{33}=-i \lambda H_{n+1}^{(2)}\left(\gamma_{s} R\right)$.

After some manipulations, it follows that

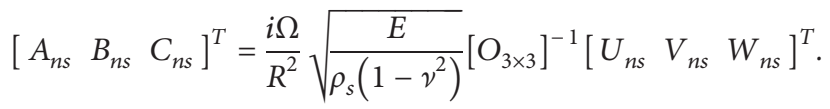

The equation of motion of the coupled system can be obtained by combining equation (24) and equation (26):

$$
\left\{\left[L_{3 \times 3}\right]+i \frac{\Omega}{h} \sqrt{\frac{1-v^{2}}{\rho_{s} E}}\left[Q_{3 \times 3}\right]\left[O_{3 \times 3}\right]^{-1}\right\}\left[\begin{array}{lll}
U_{n s} & V_{n s} & W_{n s}
\end{array}\right]^{T}=\frac{\left(1-v^{2}\right)}{E h}\left[\begin{array}{lll}
0 & 0 & F
\end{array}\right]^{T} .
$$

2.4. The Input Power Flow in the Coupled System. In order to introduce the external excitation force into the motion equations, Fourier transform is needed for the force and shell displacement.
The Fourier transform and that of differential form of variable function used in the derivation and calculation of the relevant formulas in this paper are defined, respectively, as follows: 


$$
\begin{aligned}
& \left\{\begin{array}{l}
\tilde{f}\left(k_{n s}\right)=\frac{1}{2 \pi} \int_{-\infty}^{\infty} f(x) \exp \left(-i k_{n s} x\right) \mathrm{d} x, \\
f(x)=\int_{-\infty}^{\infty} \tilde{f}\left(k_{x}\right) \exp \left(i k_{n s} x\right) \mathrm{d} k_{n s},
\end{array}\right. \\
& \left\{\begin{array}{l}
\left(i k_{n s}\right)^{n} \tilde{f}\left(k_{n s}\right)=\frac{1}{2 \pi} \int_{-\infty}^{\infty} f^{(n)}(x) \exp \left(-i k_{n s} x\right) \mathrm{d} x, \\
f^{(n)}(x)=\int_{-\infty}^{\infty}\left(i k_{n s}\right)^{n} \tilde{f}\left(k_{n s}\right) \exp \left(i k_{n s} x\right) \mathrm{d} k_{n s} .
\end{array}\right.
\end{aligned}
$$

Therefore, the external force and displacement components have the form of

$$
\begin{gathered}
\widetilde{F}=\frac{1}{2 \pi R} F_{0} \cos n \theta \exp (i \omega t) \\
\left\{\begin{array}{l}
\widetilde{U}=\frac{1}{2 \pi} \int_{-\infty}^{\infty} \sum_{n=0}^{\infty} \sum_{s=1}^{\infty} U_{n s} \cos n \theta \exp \left(\mathrm{i} \omega t-\mathrm{i} k_{n s} x\right) \mathrm{d} k_{n s} \\
\widetilde{V}=\frac{1}{2 \pi} \int_{-\infty}^{\infty} \sum_{n=1}^{\infty} \sum_{s=1}^{\infty} V_{n s} \sin n \theta \exp \left(\mathrm{i} \omega t-\mathrm{i} k_{n s} x\right) \mathrm{d} k_{n s} \\
\widetilde{W}=\frac{1}{2 \pi} \int_{-\infty}^{\infty} \sum_{n=0}^{\infty} \sum_{s=1}^{\infty} W_{n s} \cos n \theta \exp \left(\mathrm{i} \omega t-\mathrm{i} k_{n s} x\right) \mathrm{d} k_{n s} .
\end{array}\right.
\end{gathered}
$$

Substituting equations (29) and (30) into equation (27) results in

$$
\left\{\left[L_{3 \times 3}\right]+i \frac{\Omega}{h} \sqrt{\frac{1-v^{2}}{\rho_{s} E}}\left[Q_{3 \times 3}\right]\left[O_{3 \times 3}\right]^{-1}\right\}\left[\begin{array}{lll}
\widetilde{U}_{n s} & \widetilde{V}_{n s} & \widetilde{W}_{n s}
\end{array}\right]^{T}=\left[\begin{array}{lll}
0 & 0 & \frac{\Omega^{2} F_{0}}{2 \pi R \rho_{s} \omega^{2} h}
\end{array}\right]^{T} .
$$

Assuming that $\left[H_{3 \times 3}\right]=\left[L_{3 \times 3}\right]+i(\Omega / h) \sqrt{\left(1-v^{2}\right) / \rho_{s} E}$ $\left[Q_{3 \times 3}\right]\left[O_{3 \times 3}\right]^{-1}$ and $\left[I_{3 \times 3}\right]=\left[H_{3 \times 3}\right]^{-1}$, then the displacements in wave-number domain can be obtained:

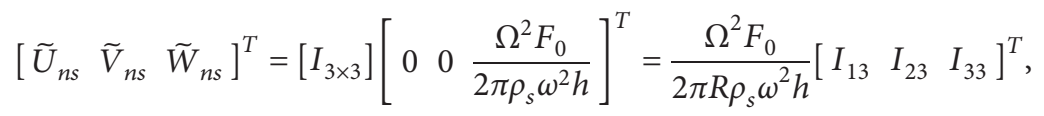

where $I_{13}=\left(\left(H_{21} H_{32}-H_{22} H_{31}\right) / \operatorname{det}|H|\right), I_{23}=\left(\left(H_{12} H_{31}-\right.\right.$ $\left.\left.H_{11} H_{32}\right) / \operatorname{det}|H|\right), I_{33}=\left(\left(H_{11} H_{22}-H_{12} H_{21}\right) / \operatorname{det}|H|\right)$.
Based on the inverse Fourier transformation of equation (32), the displacements in spatial domain can be expressed as

$$
\left[\begin{array}{c}
U_{n s}(x) \\
V_{n s}(x) \\
W_{n s}(x)
\end{array}\right]=\frac{\Omega^{2} F_{0}}{2 \pi R \rho_{s} \omega^{2} h}\left[\begin{array}{l}
\int_{-\infty}^{\infty} I_{13} \exp \left(i k_{n s} x\right) \mathrm{d} k_{n s} \\
\int_{-\infty}^{\infty} I_{23} \exp \left(i k_{n s} x\right) \mathrm{d} k_{n s} \\
\int_{-\infty}^{\infty} I_{33} \exp \left(i k_{n s} x\right) \mathrm{d} k_{n s}
\end{array}\right] .
$$

According to the definition, the input power flow of a submerged cylindrical shell can be written as

$$
P_{\text {input }}=\frac{1}{T} \int_{0}^{T} \operatorname{Re}\{F \exp (-i \omega t) \operatorname{Re}[V \exp (-i \omega t)]\} \mathrm{d} t=\frac{1}{2} \operatorname{Re}\left(F V^{*}\right)
$$


where "Re" represents the real part and “*” means taking the complex conjugate of the corresponding variable.
Substituting the radial displacement $W_{n s}(0)$ in equation (33) into equation (34) results in

$$
P_{\text {input }}=\frac{1}{2} \int_{0}^{2 \pi} \operatorname{Re}\left[F_{0} \cos (n \theta) \frac{\partial W_{n s}(0)^{*}}{\partial t}\right] R \mathrm{~d} \theta=0.5 \varepsilon_{n} \operatorname{Re}\left[i \pi F_{0} \omega W_{n s}(0)^{*}\right]
$$

where $\varepsilon_{n}=\left\{\begin{array}{ll}2, & n=0 \\ 1, & n \neq 0\end{array}\right.$.

For the purpose of facilitating the comparison, it is necessary to nondimensionalize the input power flow as

$$
\mathrm{PIN}=\frac{P_{\text {input }} \sqrt{\rho_{s} E R^{6}\left(1-\nu^{2}\right)}}{F_{0}^{2} \pi} .
$$

\section{Numerical Method and Calculating Parameters}

Only when $\left|H_{3 \times 3}\right| \neq 0$ can there be an inverse matrix $\left[I_{3 \times 3}\right]$ while $\left|H_{3 \times 3}\right|=0$ is the dispersion equation of the coupled system in free vibration. Therefore, the roots of the equation are the singular points when integrating to get the displacements in the spatial domain. These roots can be seen to have three forms [10]: purely real roots which correspond to propagating axial, torsional, and flexural type motions; purely imaginary roots which are asymptotic to the same value as those of a plate bending in the near field; and complex roots which correspond to an attenuated, near-field standing wave. Moreover, only the former ones show an impact on the value of the vibration power flow of the coupled system input from external forces [19].

As a result, the additional-damping numerical integral method is employed in the integrating process to avoid singularity. This method is to introduce damping factor $\eta$ into the shell material, and consequently the complex Young's modulus $E \prime=E(1-i \eta)$. Since the damping can effectively suppress the resonance response, the values of the integrand function at singular points on the real axis of the propagation wave change from infinity into a finite value, which means the singular point of the integrand function deviates from the real axis, so the generalized integration along the real axis can proceed smoothly.

The integral interval of equation (33) is $[-\infty,+\infty]$, which cannot be achieved in numerical integration and must be truncated. In this paper, convergence analyses are applied to obtain an alternative finite interval $[-a, a]$. If the difference rate between the integral values in the range of $[-a, a]$ and $[-0.5 a, 0.5 a]$ is within $0.5 \%$, it can be considered that the truncated interval $[-a, a]$ meets the accuracy requirements from the perspective of convergence. Then, with the interval $[-a, a]$ subdivided, ten-point Gaussian integral method is introduced and high precision solutions can be obtained.

Input vibration power flow curves are obtained for a shell made of steel immersed in viscous fluids with the amplitude of the circumferential-distributed line force
$F_{0}=1 \mathrm{~N} / \mathrm{m}$. The shell dimensions and material as well as some physical parameters of the fluid are given in Table 1.

In order to study the influence of shear and the expansive coefficients of viscosity $\mu$ and $\mu_{b}$ on the characteristics of input vibration power flow respectively, the following three sets of data are selected and referred to as Case 1, Case 2, and Case 3 [20], as listed in Table 2.

\section{Contrast and Verification}

In the previous vibroacoustic research, the acoustic medium is always considered as ideal fluid with fluid viscosity ignored. That is to say, that the shear coefficient of viscosity $\mu$ and expansive coefficient of viscosity $\mu_{b}$ are eliminated and there are only longitudinal waves considered in the ideal fluid field. This means that the velocity continuity conditions on the boundary layer only exist in the radial direction and all the algebraic terms relating to transverse vector components $\psi$ are equal to zero. However, when substituting $\psi=0$ into equation (19), the expressions of the circumferential and axial velocity still contain the terms relating to longitudinal vector components $\varphi$, contradictory to the velocity continuity condition. The difference between the wave equations to describe ideal and viscous fluid lies not only in the coefficients of viscosity, but also in the basic assumption and establishment approach. Therefore, it does not make sense in physics to substitute $\mu=\mu_{b}=0$ into the equations above and take them as of that in ideal fluids.

When the two coefficients of viscosity $\mu$ and $\mu_{b}$ are infinitely close to zero and the calculation results converge, the influence of fluid viscosity is small enough to be neglected, so the solutions can be as approximated by using the results for the ideal fluid. After several iterations (i.e., $\mu=\mu_{b}=10^{-10} \mathrm{~kg} \cdot \mathrm{m}^{-1} \cdot \mathrm{s}^{-1}, \quad \mu=\mu_{b}=10^{-12} \mathrm{~kg} \cdot \mathrm{m}^{-1} \cdot \mathrm{s}^{-1}, \quad$ and $\left.\mu=\mu_{b}=10^{-15} \mathrm{~kg} \cdot \mathrm{m}^{-1} \cdot \mathrm{s}^{-1}\right)$, the results tend to converge, which demonstrates that the choice of $\mu=\mu_{b}=10^{-15} \mathrm{~kg} \cdot \mathrm{m}^{-1} \cdot \mathrm{s}^{-1}$ is adequate for this case. Therefore, the calculation results of $\mu=\mu_{b}=10^{-15} \mathrm{~kg} \cdot \mathrm{m}^{-1} \cdot \mathrm{s}^{-1}$ will be compared to the literature values in the ideal fluid in order to verify the accuracy of the proposed method and reliability of the numerical algorithm.

Figure 2 provides a comparison between the calculation results (CR) of $\mu=\mu_{b}=10^{-15} \mathrm{~kg} \cdot \mathrm{m}^{-1} \cdot \mathrm{s}^{-1}$ and the literature values (LV) [21] with circumferential mode number $n=0$. From the figure, it can be seen that the obtained results are in good agreement with the literature values. Thus, the method and derivation in the above sections as well as the numerical algorithm are verified. 
TABLE 1: Physical parameter of the shell and fluid.

\begin{tabular}{lccccccc}
\hline & $E\left(\mathrm{~N} \cdot \mathrm{m}^{-2}\right)$ & $v$ & $\rho_{s}\left(\mathrm{~kg} \cdot \mathrm{m}^{-3}\right)$ & $R(\mathrm{~m})$ & $h / R$ & $c(\mathrm{~m} \cdot \mathrm{s})$ & $\rho_{f}\left(\mathrm{~kg} \cdot \mathrm{m}^{-3}\right)$ \\
\hline Shell & $1.92 \times 10^{11}$ & 0.3 & 7800 & 2.54 & 0.02 & - \\
Fluid & - & - & - & & - & 1500 & 1000 \\
\hline
\end{tabular}

TABLe 2: The values of coefficients of viscosity $\mu$ and $\mu_{b}$.

\begin{tabular}{lccc}
\hline Coefficients of viscosity & Case 1 & Case 2 & Case 3 \\
\hline$\mu\left(\mathrm{kg} \cdot \mathrm{m}^{-1} \cdot \mathrm{s}^{-1}\right)$ & 0.95 & 0.95 & 0.000894 \\
$\mu_{b}\left(\mathrm{~kg} \cdot \mathrm{m}^{-1} \cdot \mathrm{s}^{-1}\right)$ & 0.95 & 0.0025 & 0.0025 \\
\hline
\end{tabular}

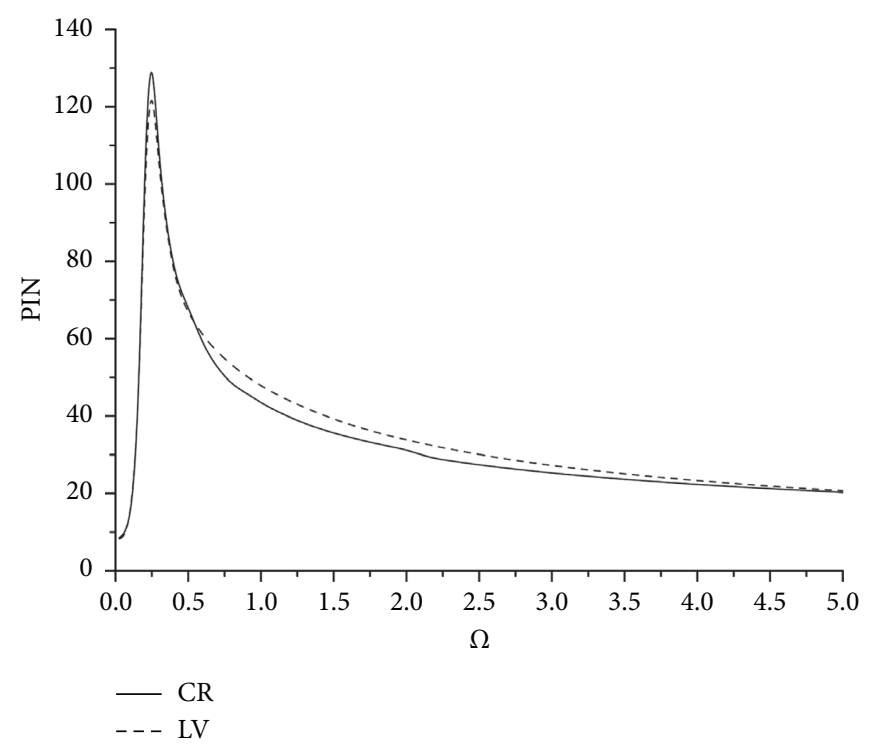

FIGURE 2: Comparison between the calculation results and the literature values $(n=0)$.

\section{The Influence of Fluid Viscosity}

The influence of fluid viscosity on input power flow of the coupled system can be demonstrated by the comparison between the calculation results of a cylindrical shell submerged in ideal fluids and that in viscous fluids under the action of the circumferential-distributional cosine-line force. Figure 3 displays the input power flow curves in the two circumstances with the parameters of Case 3 chosen and circumferential mode order from $n=0$ to $n=5$, respectively.

It is quite obvious that both the curves below have crest and share a similar variation trend. Moreover, the following conclusions can also be drawn:

(1) When the nondimensional frequency $\Omega$ is within the range of 0 to 0.2 , the viscous curves are initially lower than those in ideal fluids. Then, they tend to overlap with each other and the former even outstrip the latter while circumferential mode order $n$ increases, especially when $n>1$.

(2) The curves reach their peak when $\Omega$ is between 0.2 and 0.7 regardless of the consideration of fluid viscosity. However, the values become larger with fluid viscosity taken into account, and the difference is particularly remarkable at the peak of the curves with the maximum differential rate coming to $38 \%$. As previously mentioned, the fluid loads on axial and circumferential directions are ignored in ideal fluids, and only the radial loads on the shell are investigated. Thus, it can be seen that the input power flow of external force is promoted within this frequency band due to the consideration of transverse vibration and the dampening effect of fluid viscosity.

(3) As $\Omega$ is greater than 0.7 , the input power flow in a cylindrical shell submerged in ideal fluids is stronger than that in viscous fluids, and the maximum differential rate is around $10 \%$. It is much smaller compared to the situation in low-frequency stage and illustrates that the influence of fluid viscosity mainly concentrates in the lower band. One probable cause for this phenomenon is that the damping action of fluid viscosity on the vibration response of the shell is mainly focused in middle-high frequency band and the auxoaction mentioned above is counteracted, even transcended by the energy loss gradually.

(4) When studying the dispersion characteristics of the coupled system, a new definition of the characteristic 

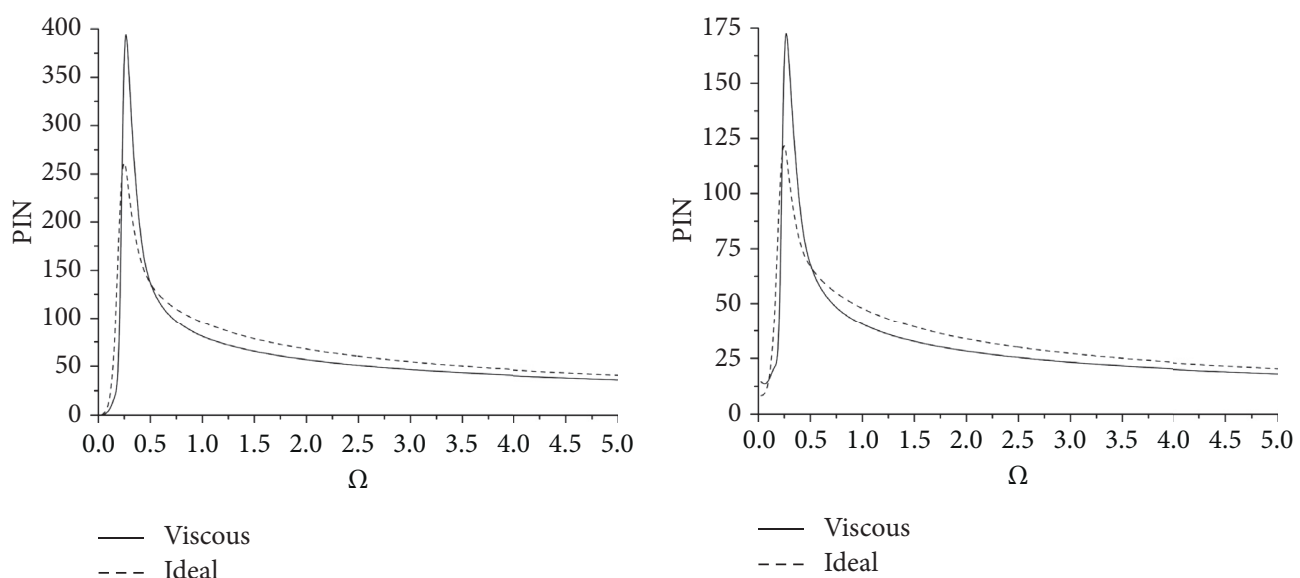

(a)

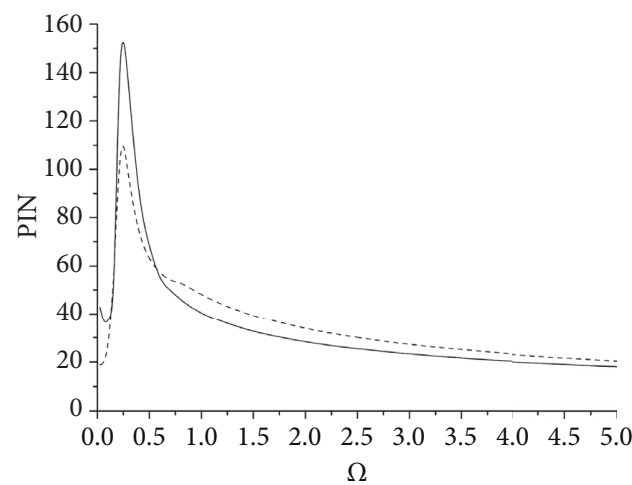

(b)

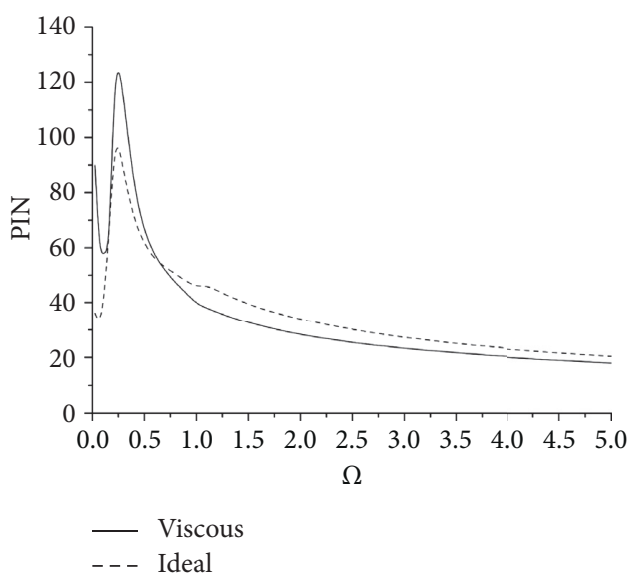

(c)
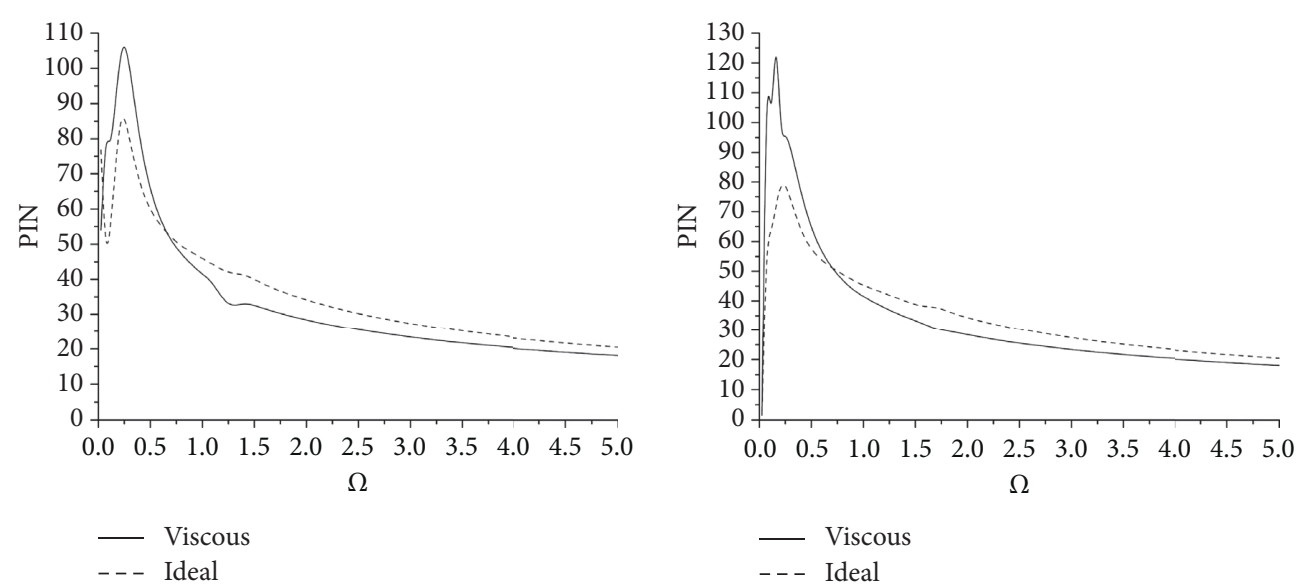

(e)

(f)

Figure 3: Input power flow in ideal and viscous fluids. (a) $n=0$. (b) $n=1$. (c) $n=2$. (d) $n=3$. (e) $n=4$. (f) $n=5$. 

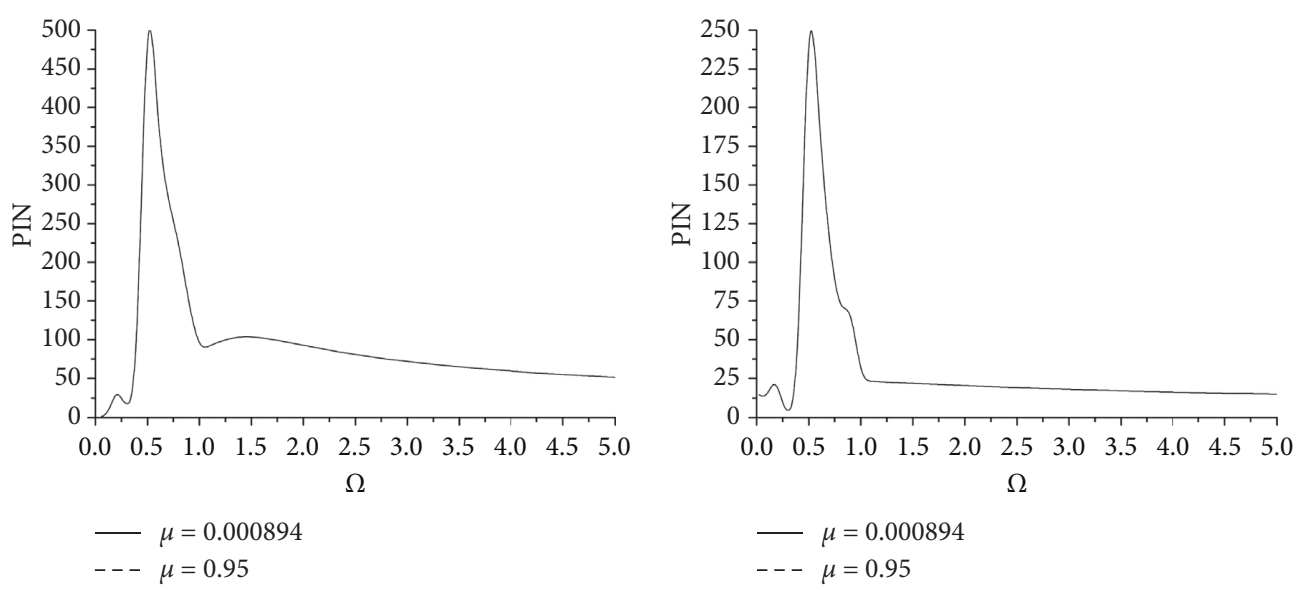

(a)
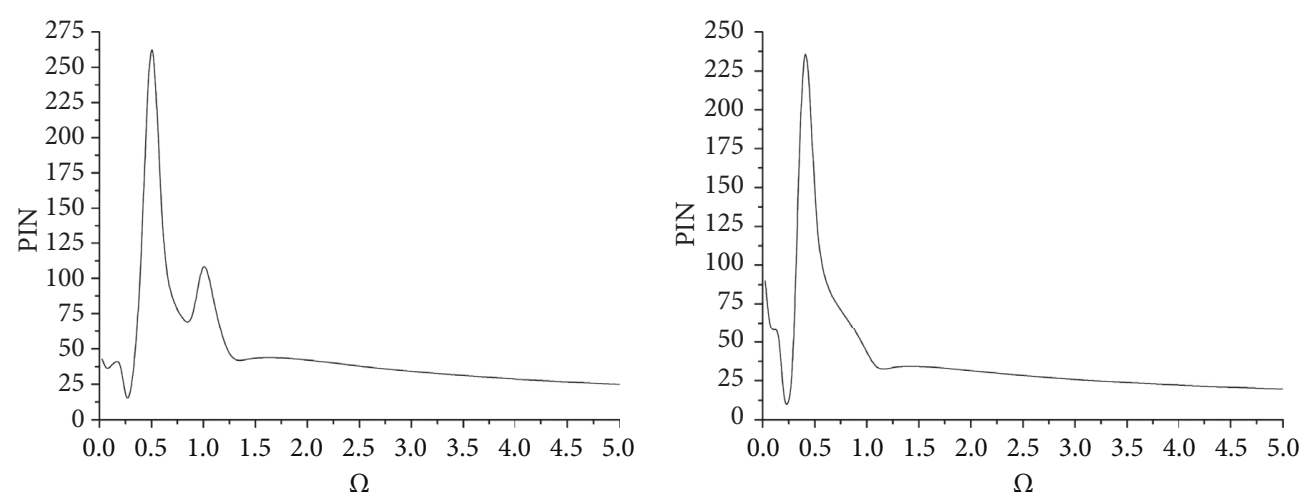

$$
\begin{aligned}
-\mu & =0.000894 \\
---\mu & =0.95
\end{aligned}
$$$$
-\mu=0.000894
$$$$
\text { - - } \mu=0.95
$$

(c)

(d)

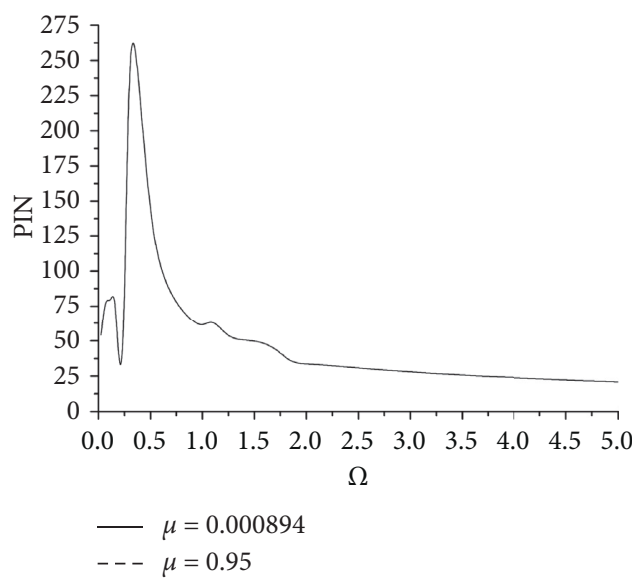

(e)

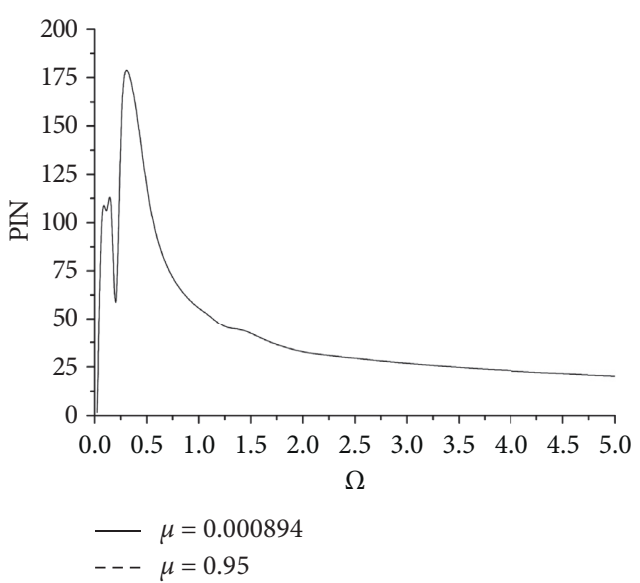

(f)

FIGURE 4: Input power flow under different shear coefficients of viscosity. (a) $n=0$. (b) $n=1$. (c) $n=2$. (d) $n=3$. (e) $n=4$. (f) $n=5$. 

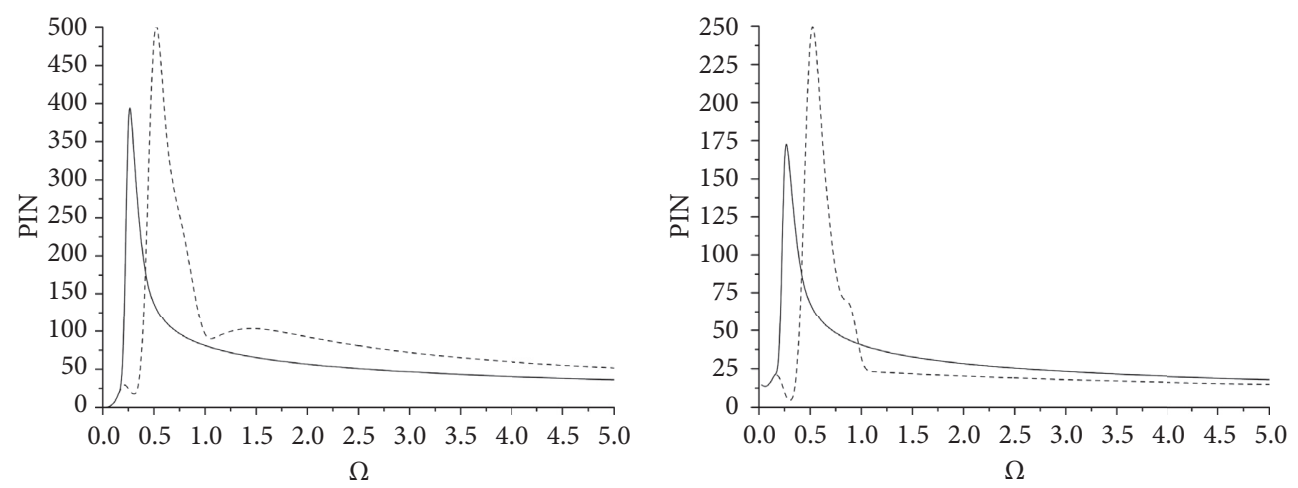

$-\mu_{b}=0.0025$

$-\mu_{b}=0.0025$

- - $\mu_{b}=0.95$

- - $\mu_{b}=0.95$

(a)

(b)
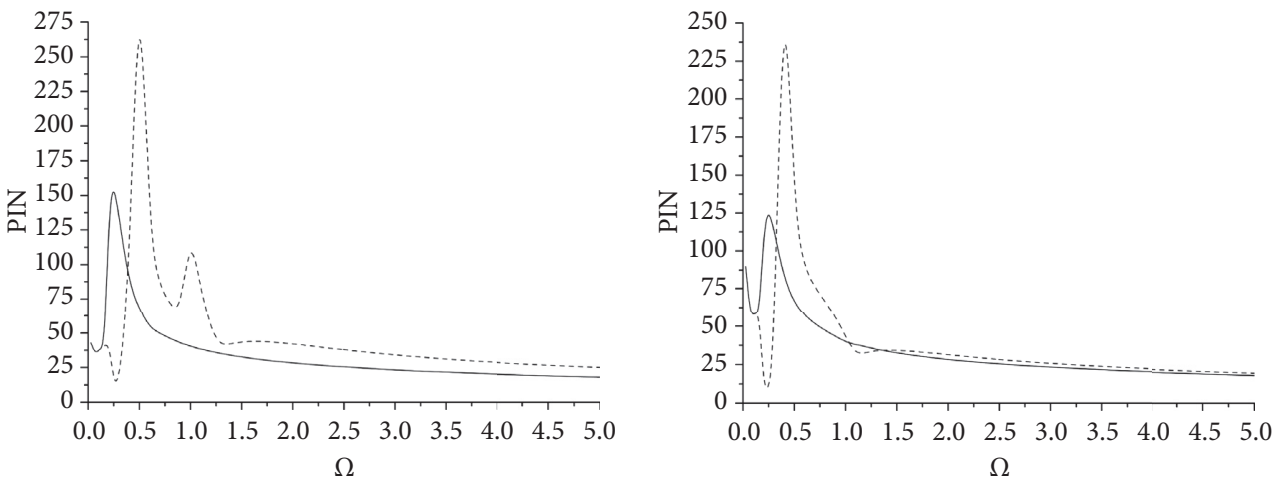

$-\mu_{b}=0.0025$

$-\mu_{b}=0.0025$

(c)

(d)

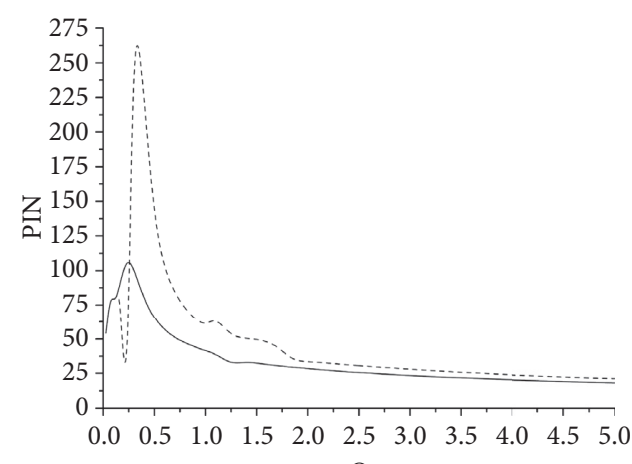

$\Omega$

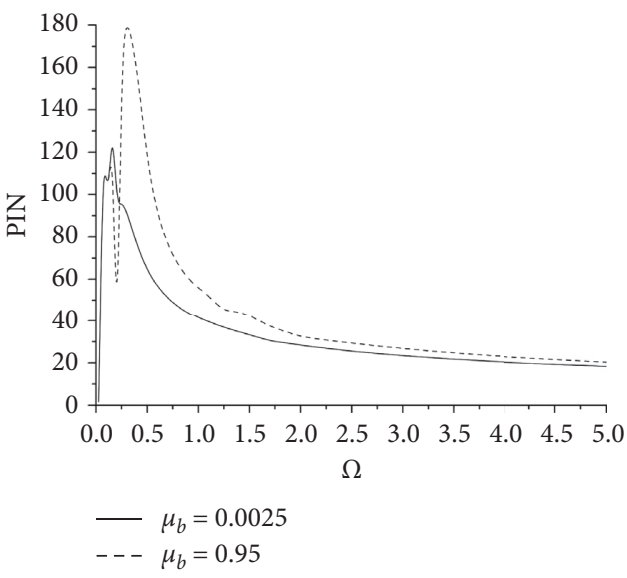

(e)

(f)

Figure 5: Input power flow under different expansive coefficients of viscosity. (a) $n=0$. (b) $n=1$. (c) $n=2$. (d) $n=3$. (e) $n=4$. (f) $n=5$. 
frequency is introduced, which means the frequency that a propagating wave just occurs [10]. Back to the curves above, the nondimensional frequencies of the crests are enlarged slightly with fluid viscosity reckoned in, and they are corresponding to the characteristic frequencies since only the propagating waves have an impact on the input power flow. The characteristic frequencies increase considering fluid viscosity, thus leading to the enlargement of the dimensionless frequencies mentioned above.

\section{The Influence of Shear Coefficient of Viscosity}

The input power flow curves of the coupled system are shown in Figure 4 when the parameters of Cases 2 and 3 are chosen, and the circumferential mode order is from $n=0$ to $n=5$, respectively. It can be observed that there are a few distinctions between the two curves in each figure. Based on the theory of Newtonian viscous liquid, the shear and expansive coefficient of viscosity are determined by the shear and volumetric strain in the fluid, respectively. As for the viscous fluid, the tangential vibration perpendicular to the propagating direction decays rapidly at the distance of one wavelength with amplitude reduced by more than 5-hundredfold, and the consequent shear strain is fairly small [22]. Therefore, the shear coefficient of viscosity $\mu$ has no discernible influence on the characteristics of the vibration power flow of the coupled system.

\section{The Influence of Expansive Coefficient of Viscosity}

Figure 5 displays the input power flow curves of the coupled system when the parameters of Cases 1 and 2 are chosen, and the circumferential mode order is from $n=0$ to $n=5$, respectively.

The values of the input power flow in the coupled system increase, especially significantly at the peak of the curves, with expansive coefficient of viscosity $\mu_{b}$ in the view of overall trends. In addition, the attenuation and propagation domains of the input power flow are alternate from the figures above. Moreover, the following phenomena can also be observed on the basis of different frequency bands:

(1) When the dimensionless frequency $\Omega$ is less than 0.2 , the input power flow curves of the coupled system almost coincide under two cases, which shows that the influence of $\mu_{b}$ in this frequency band is very weak.

(2) When $\Omega$ is between 0.2 and 0.4 , the curves of Case 2 are higher than those of Case 1 . In addition, the former have crests while the latter have troughs.

(3) When $\Omega$ is within the range from 0.4 to 1 , the curves of Case 1 all rise sharply, reach the peak position, and then drop rapidly, while the ones of Case 2 fall fast from the peak of the previous stage. In this band, the former are much higher than the latter, and the maximum gap is about $98 \%$.
(4) When $\Omega$ is greater than 1 , the changes in the curves tend to be stable under two cases. In this band, the curves of Case 1 are slightly higher than those of Case 2, and the difference between them is about $15 \%$.

According to the Newtonian viscous fluid theory, the expansive coefficient of viscosity is determined by the volume strain. In addition, the causes of the above phenomena are also related to the dispersion characteristics of the coupled system:

(1) The increase of the expansive coefficient of viscosity $\mu_{b}$ will reduce the value of the axial wave number of the coupled system, and the wave number is inversely proportional to the wave length and velocity. According to the definition, the input power flow of the coupled system is directly in proportion to the velocity, and, consequently, the values of the curves of Case 1 become larger overall, especially at the crests.

(2) Greater expansive coefficient of viscosity $\mu_{b}$ results in the increase of the occurring frequency of propagation waves, so the dimensionless frequency corresponding to the peak values of the curves increases as well.

(3) The motion form of some propagating waves changes with the expansive coefficient of viscosity $\mu_{b}$ and the circumferential mode number $n$, and greater $\mu_{b}$ and $n$ bring much more obvious influence [19]. Therefore, the attenuation and propagation domains change accordingly.

\section{Conclusion}

In this paper, the effect of fluid viscosity on the characteristics of vibration power flow in an infinite circular cylindrical shell immersed in a viscous acoustic medium is studied. Firstly, the expression of the input power flow of an infinite cylindrical shell immersed in viscous flow field under the action of the circumferential distribution line force is derived. Then, additional-damping numerical integral method and ten-point Gaussian integral method are employed to obtain the curves of input power flow curve in the coupled system. The following conclusions are drawn from the analysis:

(1) The influence of fluid viscosity on the curves of the input power flow of the coupled system is mainly concentrated in the low-frequency band $(0.2<\Omega<0.7)$.

(2) The input power flow of the coupled system increases in general after considering fluid viscosity, especially at the crests. Meanwhile, the number of the curve peaks increases, as well as the corresponding dimensionless frequencies.

(3) Shear coefficient of viscosity $\mu$ has no obvious effect on the input power flow curves of the coupled system. 
(4) Greater increase of the expansive coefficient of viscosity $\mu_{b}$ results in a larger input power flow of the coupled system, especially at the crests. In addition, the dimensionless frequencies corresponding to the curve peaks increase while the attenuation and propagation domains change accordingly.

Based on the present work, the vibroacoustic characteristics of circular cylindrical shells with finite length, which is filled with a viscous acoustic medium, will be carried out considering the external dynamic loads and the hydrostatic pressure in the following work. Furthermore, the influence of fluid viscosity in the vibroacoustic coupled system will be revealed to improve the precision of noise source identification according to Nearfield Acoustic Holography (NAH) model.

\section{Nomenclature}

\begin{tabular}{|c|c|}
\hline$\omega:$ & Circular frequency \\
\hline$F_{0}:$ & $\begin{array}{l}\text { Amplitude of the circumferential-distributed } \\
\text { line force }\end{array}$ \\
\hline : & $\delta$ function \\
\hline$U, V, W:$ & $\begin{array}{l}\text { Displacements of shell in the } x_{-}^{-}, \theta-\text {, and } r \text { - } \\
\text { direction, respectively }\end{array}$ \\
\hline$U_{n s}, V_{n s}$ & Amplitudes of $U, V, W$, respectively \\
\hline$\widetilde{U}_{n s}^{n s}, \tilde{V}_{n s}$ & $\begin{array}{l}\text { Expression of } U, V, W \text { in wave-number } \\
\text { domain, respectively }\end{array}$ \\
\hline$n:$ & Circumferential mode order \\
\hline & $\begin{array}{l}\text { Serial number of solutions of the dispersion } \\
\text { equation in axial wave numbers }\end{array}$ \\
\hline$P_{x}, P_{\theta}, P_{r}:$ & $\begin{array}{l}\text { Equivalent loads on the thin-walled cylindrical } \\
\text { shell applied by the medium in the } x-, \theta-\text {, and } r \text { - } \\
\text { direction, respectively }\end{array}$ \\
\hline$f:$ & Vibrational frequency \\
\hline$\Omega:$ & Nondimensional frequency of the elastic shell \\
\hline$\lambda:$ & Nondimensional axial wave number \\
\hline$u, v, w:$ & $\begin{array}{l}\text { Velocity components of the waves in } x^{-}, \theta_{-} \text {, and } \\
r \text {-direction, respectively }\end{array}$ \\
\hline$\sigma_{r}, \sigma_{\theta}, \sigma_{r}:$ & $\begin{array}{l}\text { Stress components of the waves in } x-, \theta-\text {, and } r \text { - } \\
\text { direction, respectively }\end{array}$ \\
\hline E: & Young's modulus of the shell \\
\hline$E^{\prime}:$ & Complex Young's modulus of the shell \\
\hline$v:$ & Poisson's ratio of the shell \\
\hline$\rho_{s}:$ & Material density of the shell \\
\hline$R:$ & Shell's mean radius \\
\hline$h:$ & Thickness of the shell \\
\hline$\rho_{f}$ : & Fluid density \\
\hline$c_{f}:$ & $\begin{array}{l}\text { Ideal speed of sound evaluated at ambient } \\
\text { conditions }\end{array}$ \\
\hline$p:$ & Sound pressure \\
\hline $\mathbf{u}_{f}$ & Fluid velocity vector \\
\hline$\varphi, \psi:$ & $\begin{array}{l}\text { Superposition of longitudinal and transverse } \\
\text { vector components }\end{array}$ \\
\hline & Shear coefficient of viscosity \\
\hline & Expansive coefficient of viscosity \\
\hline$k_{n s}:$ & Axial wave number \\
\hline & Complex compressible wave number \\
\hline
\end{tabular}

$k_{s}: \quad$ Complex shear wave number

$H_{n}^{(2)}$ : Cylindrical Hankel's functions of the second kind

$A_{n s}, B_{n s}, \quad$ Unknown coefficients

$C_{n s}$ :

$\Phi_{n s}, \Psi_{n s}: \quad$ Feature vectors

$F: \quad$ Force in complex form

$V$ : $\quad$ Velocity in complex form

T: $\quad$ Period

$P_{\text {input: }} \quad$ Input power flow

PIN: $\quad$ Nondimensional input power flow

$\eta$ : $\quad$ Damping factor.

\section{Data Availability}

No data were used to support this study.

\section{Conflicts of Interest}

The authors declare that there are no conflicts of interest regarding the publication of this paper.

\section{Acknowledgments}

The authors wish to express their gratitude to the Natural Science Foundation of Hainan Province of China (Grant no. 519QN188) and the National Natural Science Foundation of China (Grant no. 51709069) for their support.

\section{References}

[1] M. C. Junger, "Radiation loading of cylindrical and spherical surfaces," The Journal of the Acoustical Society of America, vol. 24, no. 3, pp. 288-289, 1952.

[2] M. C. Junger, "Vibrations of elastic shells in a fluid medium and the associated radiation of sound," Journal of Applied Mechanics, vol. 19, no. 2, pp. 439-445, 1952.

[3] W. Guo, T. Li, X. Zhu, and Y. Miao, "Sound-structure interaction analysis of an infinite-long cylindrical shell submerged in a quarter water domain and subject to a line-distributed harmonic excitation," Journal of Sound and Vibration, vol. 422, pp. 48-61, 2018.

[4] W. Guo, T. Li, X. Zhu, Y. Miao, and G. Zhang, "Vibration and acoustic radiation of a finite cylindrical shell submerged at finite depth from the free surface," Journal of Sound and Vibration, vol. 393, pp. 338-352, 2017.

[5] G. J. Zhang, T. Y. Li, and X. Zhu, "Series transformation method for the free vibration of eccentric cylindrical shell," Journal of Vibration Engineering, vol. 30, no. 6, pp. 947-954, 2017.

[6] Y. Y. Miao, T. Y. Li, X. Zhu et al., "Research on the acoustical radiation characteristics of cylindrical shells in a shallow sea," Journal of Harbin Engineering University, vol. 38, no. 5, pp. 719-726, 2017.

[7] M. Amabili and R. Garziera, "Vibrations of circular cylindrical shells with nonuniform constraints, elastic bed and added mass. Part III: steady viscous effects on shells conveying fluid," Journal of Fluids and Structures, vol. 16, no. 6, pp. 795-809, 2002.

[8] T. C. Su, "The effect of viscosity on free oscillations of fluidfilled spherical shells," Journal of Sound and Vibration, vol. 74, no. 2, pp. 205-220, 1981. 
[9] T. C. Su, "The effect of viscosity on free vibrations of submerged fluid-filled spherical shells," Journal of Sound and Vibration, vol. 77, no. 1, pp. 101-125, 1981.

[10] H. S. Chen, T. Y. Li, X. Zhu et al., "Analysis of dispersion characteristics of an infinite cylindrical shell submerged in viscous fluids considering hydrostatic pressure," Journal of Vibration and Acoustics-Transactions of the ASME, vol. 137, no. 2, pp. 1-14, 2015.

[11] S. V. Sorokin and A. V. Chubinskij, "On the role of fluid viscosity in wave propagation in elastic plates under heavy fluid loading," Journal of Sound and Vibration, vol. 311, no. 35, pp. 1020-1038, 2008.

[12] W. Flügge, Stress in Shells, Springer-Verlag, New York, NY, USA, 1973.

[13] M. Hasheminejad and T. L. Geers, "Modal impedances for two spheres in a thermoviscous fluid," The Journal of the Acoustical Society of America, vol. 94, no. 4, pp. 2205-2214, 1993.

[14] S. M. Hasheminejad and N. Safari, "Acoustic scattering from viscoelastically coated spheres and cylinders in viscous fluids," Journal of Sound and Vibration, vol. 280, no. 1-2, pp. 101-125, 2005.

[15] P. M. Morse and K. U. Ingard, Theoretical Acoustics, McGrawHill, New York, NY, USA, 1968.

[16] Z. Y. He, Hydrodynamic Wave Propagation Problems, National Defence Industry Press, Beijing, China, 1983.

[17] W. H. Lin and A. C. Raptis, "Acoustic scattering by elastic solid cylinders and spheres in viscous fluids," The Journal of the Acoustical Society of America, vol. 73, no. 3, pp. 736-748, 1983.

[18] W. H. Lin and A. C. Raptis, "Sound scattering from a thin rod in a viscous medium," The Journal of the Acoustical Society of America, vol. 79, no. 6, pp. 1693-1700, 1986.

[19] M. B. Xu, X. M. Zhang, and W. H. Zhang, "Input vibration power flow and its transmission in a fluid-filled shell," Acta Acoustica, vol. 24, no. 4, pp. 391-399, 1999.

[20] N. B. Vargaftik, Handbook of Physical Properties of Liquids and Gases, Springer, Berlin, Germany, 1983.

[21] Z. Z. Liu, T. Y. Li, J. J. Zhang et al., "The effect of hydrostatic pressure fields on the dispersion characteristics of fluid-shell coupled system," Journal of Marine Science and Application, vol. 9, no. 4, pp. 129-136, 2010.

[22] M. B. Vinogradova, O. V. Rudenko, and A. P. Sukhorukov, The Wave Theory, Nauka Publishers, Moscow, Russia, 1990. 